\title{
Rationalizing a Home Terabyte Server
}

\section{geck@home}

Mache Creeger, Emergent Technology Associates

\author{
Self-indulgent, \\ OR A VIEW OF \\ THE FUTURE?
}

ith 1 TB of RAID 5 storage, most of my friends believe I have really gone off the deep end with my home server. They may be right, but as in most things in life, I have gotten to this point through a rational set of individual upgrades all perfectly reasonable at the time. Rather than being overly indulgent to my inner geek, am I an early adopter of what will be the inevitable standard for home IT infrastructure? Here is my story; you be the judge.

\section{A SIMPLE ROUTER}

Eight years ago, my employer at the time graciously funded cable-modem broadband Internet service at my home so I could work from there. The base service supported only one PC. Because my wife and son each had a PC, I needed a way to share Internet access-a router to share Internet service and a home LAN to distribute network traffic.

Using PC parts, I assembled a router out of an old 486 computer with two Ethernet cards. I loaded Red Hat Linux 5.1 and configured ipchains for NAT (Network Address Translation). Everything was linked up to a 10BaseT hub. What resulted was a forerunner of the retail appliance-based router/switches available today from companies such as Linksys and D-Link.

\section{SERIOUS INTERNAL INFRASTRUCTURE}

Feature creep inevitably occurred. Since the server ran a general-purpose operating system continuously, it seemed reasonable to add more infrastructure functions. I configured Samba to make server storage accessible to the PCs on the LAN and increased disk capacity. On a roll, I then added MAC-based static allocation of DHCP addresses for the LAN PCs, along with a caching DNS and an NTP (Network Time Protocol) server.

It became more and more evident that I needed better security, so I incrementally enhanced the script for my ipchains (and later iptables) to provide the functions of a serious firewall. Starting with a base-level script that I found on the Internet, I continually tinkered, squeezing out more and more functionality. The script's size ballooned to 23 pages.

\section{MAKING THE SERVER INTERNET ACCESSIBLE}

Next I focused on making the server accessible from the outside Internet. The

WAN IP address was allocated dynamically from the ISP via DHCP, and it changed frequently. When traveling, I needed a way to access LAN resources at home from my laptop over the Internet. I wanted a fully accessible Web server, an FTP upload and download server, and the ability to use Symantec's pcAnywhere to directly control LAN-attached PCs (mostly to fix the regular disasters that occurred on my wife's and son's machines).

By placing a small DDNS (dynamic DNS) client on the server, I had a DNS name guaranteed always to resolve to the current server IP address. I installed the Apache Web server, the vsftpd (very secure FTPD) FTP server, and modified the iptables firewall script to port forward pcAnywhere traffic to its corresponding LAN-based PC.

\section{OFFLOADING TOA ROUTER APPLIANCE}

At this point, I had a miniature version of what a small to medium-size company would use for its IT infrastructure without the requisite full-time staff. Keeping the server configured and updated properly became more and more of a challenge. Software and hardware upgrades inevitably brought misconfigurations and other random problems. System crashes brought my wife and son into my office to glare at me as I feverishly worked to bring the server and our Internet access back online.

After the requisite number of kicks in the head from my family, my next effort was to offload some of the server's mission-critical functions onto a retail router/ switch appliance. I purchased a Linksys wireless router and replaced its firmware with a third-party version expanding overall functionality. Now I had much of the router and iptables firewall functionality residing on a small, low-power device that was easier to maintain. FTP, Web, and Samba services remained on the server.

\section{XIO CONTROL OF CHILD DISTRACTIONS}

All my good efforts to provide my wife and especially my 
son a rich computational environment were now being recognized. Given that my son had his own computer and a broadband Internet connection, he was very quickly becoming addicted to video games and random Internet browsing.

In her own sweet way, my wife explained to me that I needed to correct this problem quickly. More technology was needed to correct the problem of too much technology for my son. Using X10 lighting and appliance control modules, I set up password-protected and Web-enabled controls that cut video to the TVs and power to my son's computer on command and/or on a predefined schedule. Domestic harmony was soon back in balance.

\section{IMPLEMENTING MYTHTV}

My family then asked to be able to record and play back TV shows using Tivo. I did not want to pay for a Tivo unit and subscription and felt it was duplicated in what I had built in my home server. At that point I had two 200 GB drives of storage formatted in a striped disk array (RAID 0 ) and many files shared between all the machines on the LAN. I had upgraded the hardware multiple times and currently had an AMD Athlon XP 2200+ running with $757 \mathrm{MB}$ of memory.
I added more disks to the server, bringing the total number of $200 \mathrm{~GB}$ drives to six for a total of $1 \mathrm{~TB}$ of formatted RAID 5 storage. I added a used Nvidia FX 5500 graphics card and a new Hauppauge WinTV-PVR-500 video capture card. Then I installed MythTV.

Surprisingly, installing MythTV was relatively easy. Precompiled packages were available on public repositories, and Web-based step-by-step instructions were readily available. It took me one weekend from start to finish. With MythTV's free EPG (electronic programming guide) from Zap2it.com, my wife and son have a user-friendly Web interface in which to record TV and play back video on either a computer or a specific TV channel. With recording done at approximately 2 GB per hour, $1 \mathrm{~TB}$ seems to be an adequate amount of storage for home use, with approximately one-half of the available space in use at the time of this writing. The interface has a rich set of recording semantics, so my wife and son can capture as many of their favorite shows as they want. With two tuners, the PVR-500 rarely experiences conflicts in recording selections.

SO, AM I CRAZY?

Am I being self-indulgent, or is this a view of the world 
to come? Clearly, during my odyssey I discovered a need for sharing broadband Internet access through a router. This has been validated by the router/switch appliance marketplace. What else have I discovered?

- Home IT services that are not defined by what you find in a commercial IT environment. In this regard I would say that while I found value in a home FTP and Web server, its value to the overall family has been minimal.

- X10 control over video and outlet power to my son's computer has clearly been a big hit with my wife. My son hates it. It clearly addresses a common need for families with children and PC/TV management problems. We have since shown the system to several friends, who especially like the password-protected, browser-based GUI that is accessible both on the internal LAN or from any point on the Internet. No one, however, has attempted to replicate my efforts because of its perceived overall complexity.

- MythTV video capture and playback has also been a big hit. My son and wife are now confirmed viewers of nontime-based TV content.

What projects will I attempt next?

- An IPsec (IP security) gateway to allow my laptop to log in from the Internet as a peer computer on my LAN.
- The integration of all telephone services into a single gateway to include POTS (plain old telephone service) and VoIP (voice over IP).

So, is there a reason for a home server? I believe there is. Stripping away the mountains I climbed because I could from the mountains I climbed to provide value to my family, I have recognized that home IT services are not a replication of commercial ones. Services such as Internet broadband sharing, X10 power and video control, and MythTV are all viable utilities for my family. So the answer to my previous question is that my server is both self-indulgent and a view of what may occur in the future. But make no mistake, if it weren't self-indulgent, it would not have been anywhere near as fun. $Q$

\section{LOVE IT, HATE IT? LET US KNOW}

feedback@acmqueue.com or www.acmqueue.com/forums

MACHE CREEGER (mache@creeger.com) is a 30-year technology industry veteran based in Silicon Valley. He is the principal of Emergent Technology Associates, marketing and business development consultants to technology companies worldwide.

() 2006 ACM 1542-7730/06/0900 $\$ 5.00$ 